www.nature.com/pj

\title{
Self-assembled porous templates allow pattern transfer to poly(dimethyl siloxane) sheets through surface wrinkling
}

\author{
Hiroshi Yabu ${ }^{1,2}$, Yuta Saito ${ }^{3}$, Yuki Nakamichi ${ }^{3}$, Yuji Hirai ${ }^{1}$, So Fujinami ${ }^{4}$, Ken Nakajima ${ }^{4}$, Toshio Nishi $^{4}$ \\ and Masatsugu Shimomura ${ }^{1,4}$
}

Euler buckling forms surface microwrinkles ranging in size from sub-micrometers to tens of micrometers as a result of the modulus difference between the surface of the material and the material itself. When the buckling occurs in the limited areas smaller than the wrinkle wavelength, the shape of the buckled surface deforms into concave dimples or convex lenses. The shape of the buckled surface can be controlled by changing the modulus of poly(dimethylsiloxane). Hierarchical patterning can also be achieved by using a photomask. This surface wrinkling process provides a novel way to nano- and micropattern soft substrates, including elastomers and gels.

Polymer Journal (2012) 44, 573-578; doi:10.1038/pj.2012.43; published online 4 April 2012

Keywords: pattern transfer; PDMS; surface wrinkling; UV-O ${ }_{3}$

\section{INTRODUCTION}

Strong demand for the miniaturization of surface patterns has driven the development of novel fabrication technologies. Nanoimprint lithography makes it possible to realize a nanoscale surface pattern by stamping a patterned solid mold onto a melt of thermoplastic polymer or a photocurable resist. ${ }^{1}$ Xia and Whitesides $^{2}$ have reported several processes for surface pattern transfer using poly(dimethylsiloxane) (PDMS) elastomer molds; this technique is called 'soft lithography'. By using PDMS molds, the surface patterns of the PDMS elastomer mold can be easily transferred to the surface of another substrate by using polymers, ${ }^{3}$ silicates ${ }^{4}$ and so on. ${ }^{5}$ In soft lithography, microcontact printing is one of the most well-known patterning processes that facilitate the formation of thiol patterns on a gold substrate. ${ }^{6}$ After etching of the bare gold surface, gold patterns can be formed. In this process, thiol ink has an important role in the pattern transfer process. We have also reported that surface patterns can be transferred onto polymer materials by using a PDMS elastomer swollen with solvent. ${ }^{7}$ Liquid solvent softens the polymer surface, and the PDMS mold engraves the patterns onto the surface of the polymer materials. Grzybowski et al. ${ }^{8-10}$ reported that metal nanostructures can be patterned by placed hydrogels containing an etching agent on a metal surface. These examples show that chemical reactions, degradation, swelling, adsorption and so on, which deform the surface structures of materials, can be used for patterning the surface of materials.
Euler buckling is one of the universal phenomena that occurs when a stiff skin layer is formed on a soft substrate. ${ }^{11}$ Because of Euler buckling, surface wrinkling occurs on soft substrates. There are some reports, both theoretical and experimental, describing the formation of microwrinkles originating from Euler buckling. Whitesides et al. ${ }^{11}$ reported the formation of microwrinkles when forming a metal layer on a patterned PDMS surface. Rogers et al. ${ }^{12}$ showed the buckling of GaAs ribbons and its application for electronics. Stafford et al. ${ }^{13}$ showed that microwrinkles formed when grafting polymer brushes onto PDMS surfaces. Many other studies have reported the formation of microwrinkles and their applications. ${ }^{14-19}$ It has been reported that periodic microwrinkles are formed when the surface of the PDMS elastomer is oxidized by $\mathrm{O}_{2}$ plasma or $\mathrm{UV}-\mathrm{O}_{3}$ treatments owing to the difference in Young's elastic modulus between the oxidized surface layers and bulk PDMS. ${ }^{20}$ The periodicity of the microwrinkles can be controlled by changing the thickness and elastic modulus of the oxidized skin layer and PDMS, respectively. Kim et al. ${ }^{4}$ have reported that instead of microwrinkle formation, a convex microlens can be formed on a stretched PDMS sheet by introducing UV-O $\mathrm{O}_{3}$ through a porous mask following release of the stretching force. Chan and Crosby $^{21}$ showed the confinement effect on the surface wrinkling of PDMS from introducing $\mathrm{UV}^{-\mathrm{O}_{3}}$ through a mask with various pore sizes. They also show that the surface wrinkles disappeared when the size of the oxidized area was smaller than the persistent length of buckling. ${ }^{22}$ We also observed the same confinement effect on the

\footnotetext{
${ }^{1}$ Institute of Multidisciplinary Research for Advanced Materials (IMRAM), Tohoku University, Sendai, Japan; ${ }^{2}$ Precursory Research for Embryonic Science and Technology (PRESTO), Japan Science and Technology Agency (JST), Sendai, Japan; ${ }^{3}$ Graduate School of Engineering, Tohoku University, Sendai, Japan and ${ }^{4}$ WPI-Advanced institute for Materials Research (AIMR), Tohoku University, Sendai, Japan

Correspondence: Dr H Yabu, Institute of Multidisciplinary Research for Advanced Materials (IMRAM), Tohoku University, 2-1-1, Katahira, Aoba-ku, Sendai 980-8577, Japan. E-mail: yabu@tagen.tohoku.ac.jp

Received 28 November 2011; revised 27 January 2012; accepted 19 February 2012; published online 4 April 2012
} 
surface wrinkles of the metal dots formed on a shrinkable polymer substrate. $^{23-27}$ The persistent length $(\zeta)$ of one-dimensional buckling is calculated by the following equation:

$$
\zeta=\lambda_{0}\left(\frac{1}{2 \epsilon^{1 / 2}}+2 \eta^{1 / 3}\right)
$$

Here, $\lambda_{0}$ is the buckling wavelength of a semi-infinite surface, $\epsilon$ is the strain of the local region, and $\eta$ is the moduli-mismatch ratio. The length scale $\zeta$ is a critical parameter in determining the morphology of the buckling structures. Typically, the $\zeta$ of an oxidized layer on a PDMS surface is approximately $50 \mu \mathrm{m}$. For dimensions D much greater than the persistence length, the wrinkles persist radially inward to $\zeta$. Beyond this length, they interact with adjacent wrinkles. However, if the pore size of the mask $(D)<\zeta$, the surface pattern can be transferred to the PDMS surface by $\mathrm{UV}-\mathrm{O}_{3}$ exposure, and two types of buckled structures are observed: the microlens pattern and the dimple pattern. ${ }^{22}$

In this paper, we show a novel surface patterning method based on the buckling of PDMS elastomers. A porous template was prepared by using self-organized honeycomb films or colloidal crystals. After transferring the template onto PDMS films with different elastic moduli, the porous patterns were transferred onto the PDMS surface as dimples or lenses via the introduction of $\mathrm{UV}-\mathrm{O}_{3}$ onto the PDMS surface through the template pores. Hierarchical patterning of the dimple structure was also shown. The surface patterns and the surface modulus are discussed based on the results of atomic force microscopy (AFM) measurements.

\section{MATERIALS AND METHODS}

\section{Preparation of porous templates:}

Honeycomb-patterned polymer films were prepared by a simple casting process under humid conditions. ${ }^{28}$ An amphiphilic copolymer (1) was synthesized according to the procedure outlined in the literature Chart $1 .{ }^{29}$ Polystyrene (PS) and 1 were dissolved in chloroform to form a $10 \mathrm{mg} \mathrm{ml}^{-1}$ solution. The solution was cast onto a glass substrate, and the solvent was evaporated by applying a flow of humid air (relative humidity $>90 \%$ ) with a velocity of $1001 \mathrm{~min}^{-1}$. After complete evaporation of the solvent, the surface structure was observed by using an optical microscope or a scanning electron microscope (SEM)

Colloidal crystal films were prepared by coating an aqueous dispersion of PS latex (diameter $=500 \mathrm{~nm}$, Duke Scientific, Waltham, MA, USA) by using a handmade apparatus described in the literature. ${ }^{30}$ Typically, the two glass slides were placed parallel with a $500 \mu \mathrm{m}$ gap, and then, the aqueous dispersion of PS latex was used to fill in the gap between the glass slides. One glass slide moved steadily at a velocity of $10 \mu \mathrm{ms}^{-1}$, and the colloidal crystal films were continuously formed from the meniscus of the aqueous dispersion formed at the edge of the glass slide. After complete evaporation of water, fcc-type colloidal crystal films were prepared. The surface structure of the film was observed by using an SEM.

\section{Experimental Details}

Preparation of PDMS sheets:. PDMS elastomer sheets having various moduli were prepared from Sylgard 184 (Dow Corning, Midland, MI, USA). The

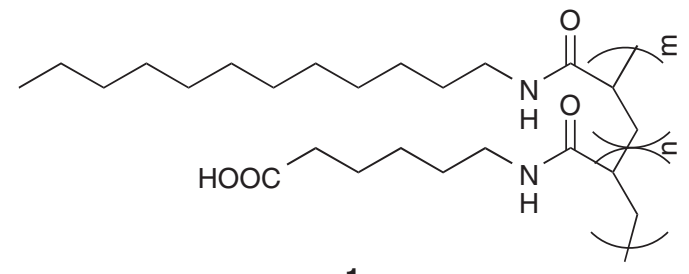

1

Chart 1 Chemical structure of polymer $1(\mathrm{~m} / \mathrm{n}=4)$ prepolymer of PDMS and the Pt catalyst were mixed and cast on a glass dish $(\phi=90 \mathrm{~mm})$. After degassing in vacuo for $1 \mathrm{~h}$, the PDMS elastomer sheets were prepared by heating the samples at 70,120 and $200^{\circ} \mathrm{C}$ for $2 \mathrm{~h}$. The mixing ratio between the PDMS prepolymer and the catalyst was changed from tento-one to forty-to-one.

Surface wrinkling. A schematic illustration of surface wrinkling is shown in Figure 1a. The PDMS elastomer sheet was washed with benzene or chloroform, and then dried at room temperature. The PDMS elastomer sheet was cut using a surgery knife and removed from the glass dish with tweezers. The PS honeycomb films were treated with $\mathrm{UV}-\mathrm{O}_{3}$ to make the film brittle by using a $\mathrm{UV}^{-\mathrm{O}_{3}}$ cleaner (OC-250615-D + A, IWASAKI, Tokyo, Japan) equipped with six $25 \mathrm{~W}$ lamps. ${ }^{31}$ The PDMS elastomer sheet was placed on the porous template upside down and pressed. Only the surface porous structure was transferred onto the PDMS elastomer after removing the PDMS elastomer from the template surface. The UV-O 3 treatment was performed for 3-10 min. After the $\mathrm{UV}-\mathrm{O}_{3}$ treatment, the porous template was removed with adhesive tape.

The same surface wrinkling process was performed by using colloidal crystal templates. After the formation of colloidal crystals, a PDMS sheet whose $\epsilon$ was $0.18 \mathrm{MPa}$ was placed on the colloidal crystal and pressed.

Hierarchical patterning was performed by placing a photomask (Edmund Scientific, New York, NY, USA) on the template-loaded PDMS sheet. After the same surface wrinkling procedures, the surface pattern was formed after removing the template.

Analysis. The surface structures of prepared samples were observed by using a digital optical microscope (VHX-1000, Keyence, Tokyo, Japan), a field emission-type scanning electron microscope (FE-SEM, S-5200, HItachi, Tokyo, Japan), an atomic force microscope (AFM, SPI400, Seiko Instruments, Chiba, Japan), and a laser scanning microscope (LSM, LEXT OLS4000, Olympus, Tokyo, Japan). Young's elastic modulus of the prepared PDMS elastomer sheets was measured by a drawing experiment. ${ }^{15}$ The edges of a PDMS specimen were fixed on the clamps of a drawing stage and a load cell (SPZ, IMADA, Toyohashi, Japan), and then the sample was stretched and the applied force and strain of the sample were measured. The PDMS elastic modulus $\epsilon$ is extracted from the mono-axial strain-stress equation $\sigma_{\mathrm{zz}}=\epsilon \bullet E$, where $\sigma_{\mathrm{zz}}$ is the axial true stress applied to the PDMS and $E$ is the axial strain of the PDMS. We have $\mathrm{dF} / S_{z}$, where $\mathrm{dF}$ is the axial resultant force applied to the PDMS and $S_{z}$ is the cross section of the PDMS specimen; $E=\delta \mathrm{l}_{0} / l_{0}$, where $l_{0}$ is the initial length of the specimen and $\delta \mathrm{l}_{0}$ is the stretch for the current axial resultant force. Thus, Young's modulus $\epsilon$ of PDMS is obtained from the following equation:

$$
\epsilon=\frac{d F_{z}}{S_{z}} \cdot \frac{l_{0}}{\delta l_{0}}
$$

The modulus of PDMS elastomer sheets before/after UV-O ${ }_{3}$ treatment was measured by using an AFM. ${ }^{32}$ By using a procedure based on a combination of Johnsone-Kendalle-Robert contact mechanics and a 'two-point method' together with AFM force volume measurements, maps of the elastic modulus of the PDMS surface were obtained. Force volume (FV) experiments were performed using a commercial AFM system (Multimode series with a NanoScope $\mathrm{V}$ controller) under ambient conditions. In the FV-mode, force curves were collected over randomly selected surface areas at a resolution of $64 \times 64$ pixels. The cantilevers used were OMCL-TR800PSA-1 (Olympus), whose nominal spring constant is $0.57 \mathrm{~N} \mathrm{~m}^{-1}$. The actual spring constant was measured by the thermal tune method with a value of the spring constant of $0.53 \mathrm{~N} \mathrm{~m}^{-1}$. The calibrated deflection sensitivity with a hard metal surface was used in the analyses.

\section{RESULTS AND DISCUSSION}

\section{Surface pattern transfer of honeycomb structures onto a PDMS} surface

Figure $1 \mathrm{~b}$ shows a typical SEM image of honeycomb films. A hexagonally arranged microporous structure was clearly observed. The pore size of the honeycomb film used in this experiment was $14 \mu \mathrm{m}$, which is smaller than the $\zeta$ of PDMS microwrinkles. The inset 

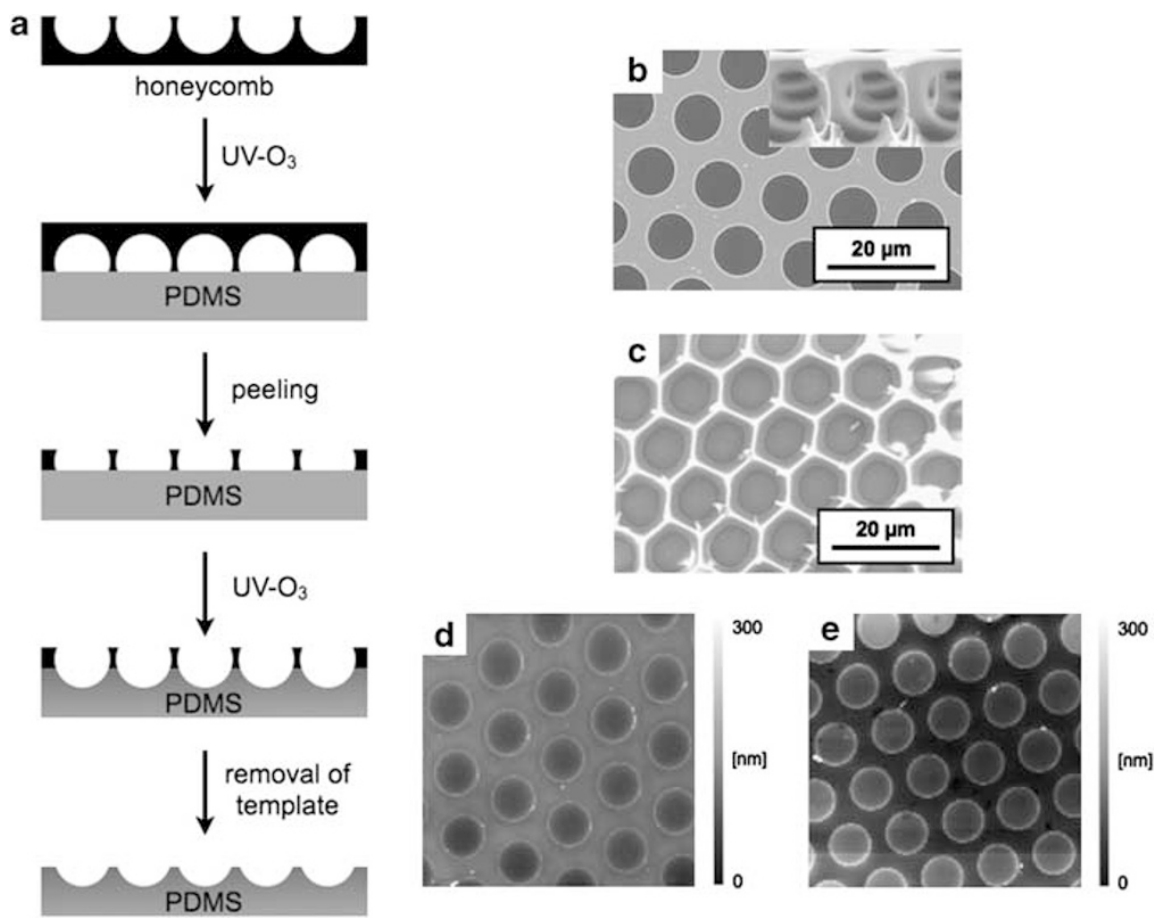

Figure 1 Schematic illustration of the surface wrinkling process (a), an scanning electron microscope (SEM) image of a honeycomb film of Polystyrene (b), an SEM image of a porous template on a poly(dimethylsiloxane) (PDMS) sheet (c), and atomic force microscopy images of a concave dimple array (d) and a convex lens array (e).

image in Figure $1 \mathrm{~b}$ shows a cross-sectional SEM image of the honeycomb film. It has a double-layered structure supported by pillars at the apexes of the honeycomb hexagons. After pressing onto the PDMS surface, the center portion of the pillar, which is mechanically the weakest portion of the pillar, was broken. Figure 1c shows an SEM image of the porous template on the PDMS sheet. The hexagonally arranged porous template was clearly observed. Figure 1d shows an AFM image of the dimple array structure prepared by a $15-\mathrm{min} \quad \mathrm{UV}_{-} \mathrm{O}_{3}$ treatment through the honeycomb template onto the PDMS sheet whose $\epsilon$ was $1.18 \mathrm{MPa}$. Concave-shaped dimples are clearly observed. In contrast, the convexshaped lenses were observed on the PDMS surface whose $\epsilon$ was $0.18 \mathrm{MPa}$ (Figure 1e).

These structural differences were caused by the $\epsilon$ difference of PDMS. Figure 2 shows plots of the respective depths and heights of dimples and lenses formed on PDMS sheets having various $\epsilon$. When $\epsilon$ is high enough, concave-shaped dimples were formed on the surface (Figure 2a). The depth of the dimple increased with decreasing $\epsilon$ until c.a. $0.5 \mathrm{MPa}$. When the $\epsilon$ decreased, the shape of the $\mathrm{UV}^{-\mathrm{O}_{3} \text {-exposed }}$ region changed from concave dimples to convex lenses (Figure $2 b$ ). The height of the lenses also increased with decreasing $\epsilon$. As reported in the literature, the surface structure transition from dimple to lens was observed under strong confinement $(D<<\zeta) .{ }^{23-26}$ According to the equation (1), the $\zeta$ of buckling increases with decreasing $\epsilon$ of PDMS. Thus, the oxidized layer on the soft PDMS substrate is under a stronger confinement field than that of the hard PDMS substrate. Because of the oxidization, the volume of the oxidized layer gradually increased, as a result, the curvature of the surface was inverted. Thus, this strong confinement makes the oxidized surface protrude.

As shown in Figure 2, the depths and heights of the respective dimples and lenses were increased with increasing $\mathrm{UV}-\mathrm{O}_{3}$ treatment

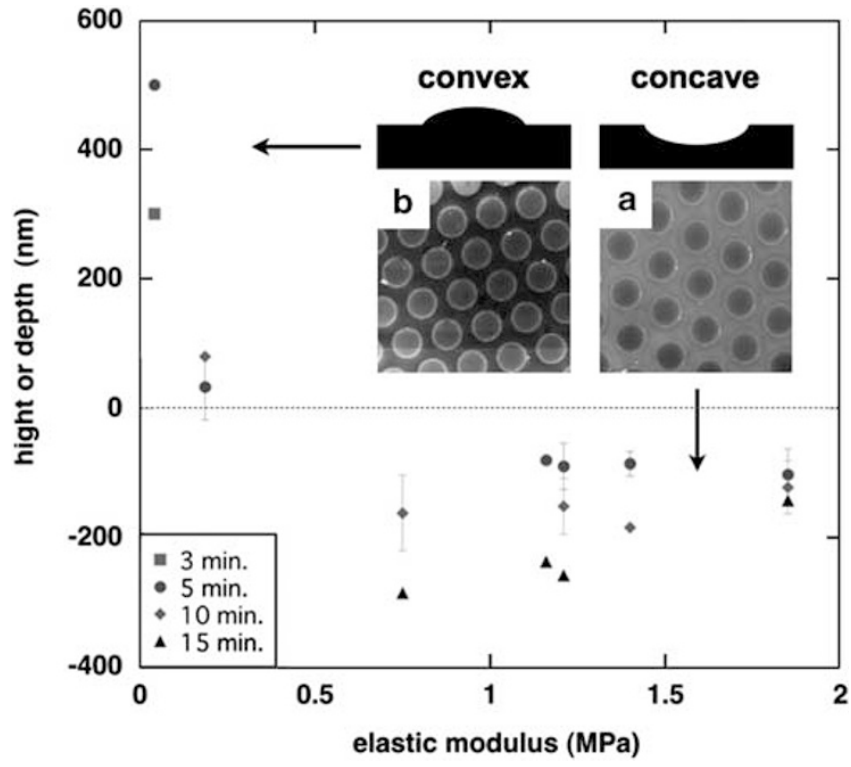

Figure 2 Relationship between the elastic modulus of poly(dimethylsiloxane) sheets and the height or depth of the concave dimples and convex lenses. The depths and heights of the dimples and lenses after $3 \mathrm{~min}(\boldsymbol{\square})$, $5 \mathrm{~min}(\bullet), 10 \mathrm{~min}(\bullet)$, and $15 \mathrm{~min}(\mathbf{\Delta})$ of $\mathrm{UV}-\mathrm{O}_{3}$ treatment.

time. Supplementary Figure S1 For example, the depths of dimples formed on a 1.8 MPa PDMS sheet decreased from 143 to $102 \mathrm{~nm}$ with increasing $\mathrm{UV}^{-} \mathrm{O}_{3}$ treatment time from 5 to $15 \mathrm{~min}$. The amplitude of the deformation depends on the difference of $\epsilon$ between the oxidized 

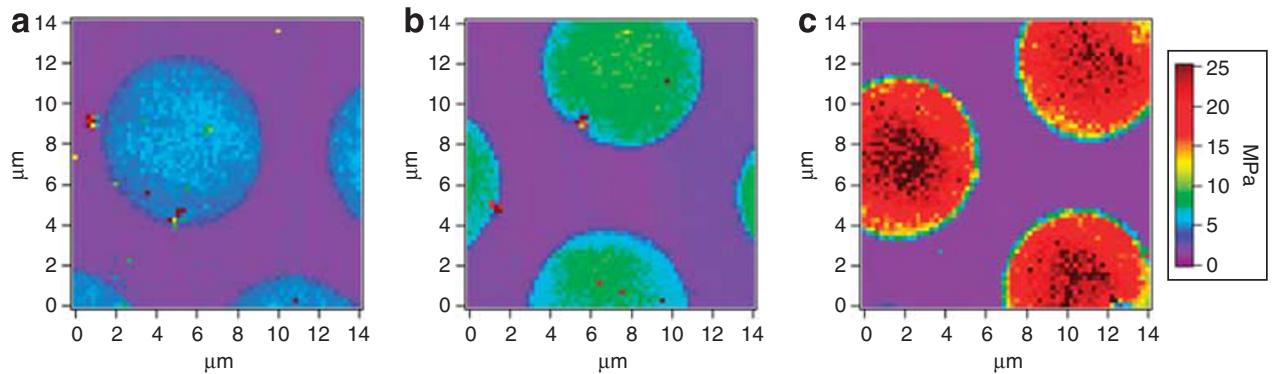

Figure 3 Elastic modulus mapping of poly(dimethylsiloxane) surfaces after $5 \mathrm{~min}$ (a), $10 \mathrm{~min}$ (b) and $15 \mathrm{~min}$ (c) of UV- $\mathrm{O}_{3}$ treatment measured by using an atomic force microscopy.

layer and the bulk PDMS. The modulus distribution on the PDMS surface was measured by FV AFM measurements. The surface modulus can be calculated from the pull-out force between an AFM cantilever and the PDMS surface. By using this method, the effect of the $\mathrm{UV}-\mathrm{O}_{3}$ exposure time on the surface modulus was observed. Figures 3a-c show two-dimensional modulus distributions at the surface of the PDMS sheets, which had a $\epsilon$ of $1.8 \mathrm{MPa}$ after the formation of dimples by $\mathrm{UV}-\mathrm{O}_{3}$ treatment for $5-15 \mathrm{~min}$. The modulus measured on the dimples increased with increasing $\mathrm{UV}-\mathrm{O}_{3}$ exposure time. In contrast, the other portion of the PDMS surface did not show any significant modulus changes compared with their original modulus. Because of the difference in the surface modulus and the bulk modulus, the depth of the dimple increased with increasing $\mathrm{UV}-\mathrm{O}_{3}$ exposure time.

Hierarchical pattering of honeycomb structures onto PDMS sheets Hierarchical patterning of the concave-shaped dimple array structure was fabricated by exposing $\mathrm{UV}-\mathrm{O}_{3}$ through a photomask onto a PDMS sheet covered with a porous template (Figure 4a). Because $\mathrm{O}_{3}$ molecules were generated by irradiation with UV light of atmospheric $\mathrm{O}_{2}$ molecules in the $\mathrm{UV}-\mathrm{O}_{3}$ chamber, the generation of $\mathrm{O}_{3}$ was prevented at the PDMS surface covered with the photomask. Figure $4 \mathrm{~b}$ shows a photograph of hierarchically patterned PDMS sheets whose $\epsilon$ was $1.4 \mathrm{MPa}$ after a 15 -min $\mathrm{UV}_{-} \mathrm{O}_{3}$ treatment. The patterns of the photomask were clearly observed. Figures $4 \mathrm{c}$ and $\mathrm{d}$ show an optical micrograph and an LSMS image of patterned PDMS surfaces, respectively. A clear boundary between the concave-shaped dimples and the nondimpled surface was observed. From these results, hierarchical patterning via a combination of the porous template and the photomask was demonstrated by using surface wrinkling. The convex-shaped lenses are also hierarchically patterned by using a photomask when the $\epsilon$ of the PDMS sheet was $0.18 \mathrm{MPa}$.

\section{Pattern transferring of colloidal crystals}

The resolution limit of the lithographic technique is an important factor for practical applications. To examine the nanoscale pattern transfer using the surface wrinkling, colloidal crystals of $500 \mathrm{~nm}$ PS particles were used as templates. Figure $5 \mathrm{a}$ shows a schematic illustration of the sample preparation procedure. Colloidal crystals of $500 \mathrm{~nm}$ PS colloidal particles were prepared and placed on a PDMS sheet $(\epsilon=0.18 \mathrm{MPa})$. After $\mathrm{UV}-\mathrm{O}_{3}$ treatment, the surface pattern was observed by using an AFM. Figure $5 \mathrm{~b}$ shows prepared surface patterns on the PDMS sheet. Hexagonally arranged dimples were formed. In this case, the UV-O ${ }_{3}$ was introduced through the gap between the colloidal crystals, and then, the hexagonal rims rose up spontaneously. Finally, the hexagonally arranged dimples were clearly observed. The a

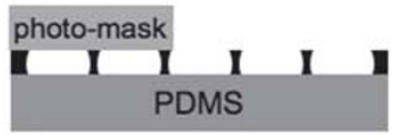

$\mathrm{UV}-\mathrm{O}_{3}$

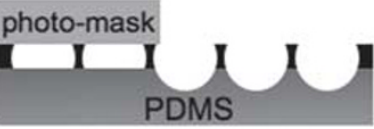

removal of photo-mask
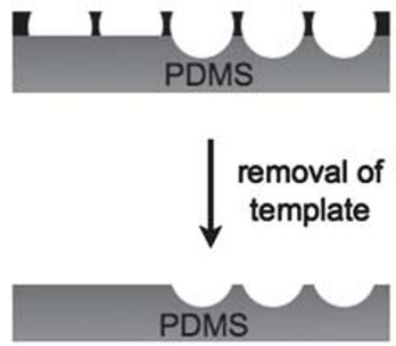

Figure 4 Schematic illustration of the hierarchical patterning process of surface wrinkling (a), a photograph (b), an optical micrograph (c) and a laser scanning microscope image (d) of a hierarchically patterned poly(dimethylsiloxane) (PDMS) sheet.

width of the rim was c.a. $100 \mathrm{~nm}$, which was smaller than the wavelength of the UV light $(\lambda=197 \mathrm{~nm})$ used to generate $\mathrm{O}_{3}$ molecules from the $\mathrm{O}_{2}$ molecules in the chamber. Because the buckling caused formation of a surface-oxidized layer via reactive $\mathrm{O}_{3}$ molecules, the resolution of the pattern transfer does not depend on the wavelength of the light. This result indicates that this surface wrinkling process can transfer a pattern that is smaller than the diffraction limit of the light.

\section{CONCLUSION}

In this paper, we show a novel pattern transfer process using Euler buckling. By using the buckling process, concave-shaped dimples or convex-shaped lenses were formed on PDMS sheets, depending on 

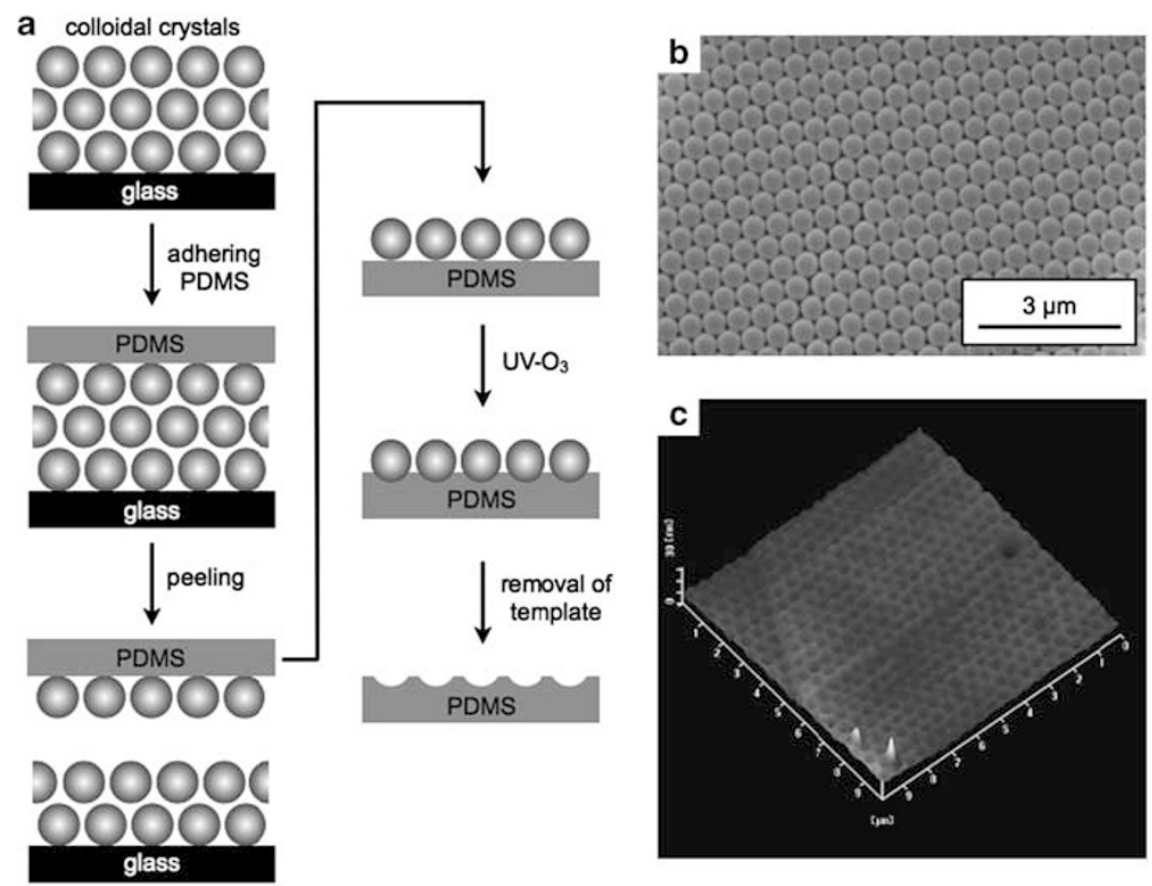

Figure 5 Schematic illustration of the surface wrinkling process using colloidal crystals (a), an scanning electron microscope image of the colloidal crystal (b), and an atomic force microscopy image of surface patterns formed on a poly(dimethylsiloxane) (PDMS) sheet (c).

the elastic modulus of the PDMS sheets. Hierarchical patterning of the PDMS surface could be achieved by using photomasks. The resolution of this process can exceed the diffraction limit of the UV light used in this experiment. This 'surface wrinkling' process can be used as a novel fabrication technique for patterning soft and brittle materials without any expensive equipment. By using this process, surface patterns can be created on various soft materials, including rubbers, gels and stretched polymer films. These patterned materials may be applicable for various photonic, electronic and biotechnological applications.

\section{ACKNOWLEDGEMENTS}

HY would like to thank Mr Shunsuke Sato for assisting with the SEM measurements. This research is partially supported by a Grant-in-Aid for Scientific Research (C), MEXT, JAPAN.

1 Guo, L. J. Nanoimprint lithography: methods and material requirements. Adv. Mater. 19, 495 (2007).

2 Xia, Y. \& Whitesides, G. M. Soft lithography. Angew. Chem. Int. Ed. Engl. 37, 551-575 (1998).

3 Xia, Y., McClelland, J. J., Gupta, R., Qin, D., Zhao, X. M., Sohn, L. L., Celotta, R. J. \& Whitesides, G. M. Replica molding using polymeric materials: a practical step toward nanomanufacturing. Adv. Mater. 9, 147-149 (1997).

4 Trau, M., Yao, N., Kim, E., Xia, Y., Whitesides, G. M. \& Aksay, I. A. Microscopic patterning of orientated mesoscopic silica through guided growth. Nature 390, 674-676 (1997).

5 King, E., Xia, Y. N., Zhao, X. M. \& Whitesides, G. M. Solvent-assisted microcontact molding: a convenient method for fabricating three-dimensional structures on surfaces of polymers. Adv. Mater. 9, 651-654 (1997).

6 Xia, Y., Qin, D. \& Whitesides, G. M. Microcontact printing with a cylindrical rolling stamp: a practical step toward automatic manufacturing of patterns with submicrometer-sized features. Adv. Mater. 8, 1015-1017 (1996).

7 Yabu, H. \& Shimomura, M. Miniaturization of surface patterns by combination of contact etching lithography and multi-step shrinking of stretched polymer films. Polym. J. 40, 534-538 (2008).

8 Smoukov, S. K., Bishop, K. J. M., Klajn, R., Campbell, C. J. \& Grzybowski, B. A Cutting into solids with micropatterned gels. Adv. Mater. 17, 1361-1365 (2005).
9 Grzybowski, B. A., Bishop, K. J. M., Campbell, C. J., Fialkowski, M. \& Smoukov, S. K. Micro-and nanotechnology via reaction-diffusion. Soft Matter 1, 114-128 (2005).

10 Campbell, C. J., Klajn, R., Fialkowski, M. \& Grzybowski, B. A. One-step multilevel microfabrication by reaction-diffusion. Langmuir 21, 418-423 (2005).

11 Boden, N., Brittain, S., Evans, A. G., Hutchinson, J. W. \& Whitesides, G. M. Spontaneous formation of ordered structures in thin films of metals supported on an elastomeric polymer. Nature 393, 146-149 (1998).

12 Sun, Y., Kumar, V., Adesida, I. \& Rogers, J. A. Buckled and wavy ribbons of GaAs for high-performance electronics on elastomeric substrates. Adv. Mater. 18, 2857-2862 (2006).

13 Huang, H., Chung, Y., Nolte, A. J. \& Stafford, C. M. Characterizing polymer brushes via surface wrinkling. Chem. Mater. 19, 6555-6560 (2007).

14 Zhang, H. L., Okayasu, T. \& Bucknall, D. G. Large area ordered lateral patterns in confined polymer thin films. Eur. Polym. J. 40, 981-986 (2004).

15 Wang, X. M. \& Grundmeier, G. Morphology and patterning processes of thin organosilicon and perfluorinated bi-layer plasma polymer films. Plasma Process Polym. 3, 39-47 (2006).

16 Suh, K. Y., Seo, S. M., Yoo, P. J. \& Lee, H. H. Formation of regular nanoscale undulations on a thin polymer film imprinted by a soft mold. J. Chem. Phys 124 , 024710 (2006).

17 Ohzono, T., Watanabe, H., Vendamme, R., Kamaga, C., Kunitake, T., Ishihara, T. \& Shimomura, M. Spatial forcing of self-organized microwrinkles by periodic nanopatterns. Adv. Mater. 19, 3229-3232 (2007).

18 Pocivavsek, L., Dellsy, R., Kern, A., Johnson, S., Lin, B., Lee, K. Y. C. \& Cerda, E. Stress and fold localization in thin elastic membranes. Science 320, 912-916 (2008).

19 Chan, E. P. \& Crosby, A. J. Spontaneous formation of stable aligned wrinkling patterns. Soft Matter 2, 324-328 (2006)

20 Chandra, D., Yang, S. \& Lin, P. C. Strain responsive concave and convex microlens arrays. Appl. Phys Lett. 91, 251912 (2007).

21 Chan, E. P. \& Crosby, A. J. Fabricating microlens arrays by surface wrinkling. Adv. Mater. 18, 3238-3242 (2006).

22 Yabu, H., Matsuo, Y., Ijiro, K., Nishino, F., Takaki, T., Kuwahara, M. \& Shimomura, M. Spontaneous formation of microwrinkles on metal microdot arrays by shrinking of thermal shrinkable substrate. ACS Appl. Mater. Inter. 2, 23-27 (2010).

23 Widawski, G., Riwaso, M. \& François, B. Self-organized honeycomb morphology of starpolymer polystyrene films. Nature 369, 387-389 (1994).

24 Maruyama, N., Koito, T., Nishida, J., Sawadaishi, T., Cieren, X., Ijiro, K., Karthaus, O. \& Shimomur, M. Mesoscopic patterns of molecular aggregates on solid substrates. Thin Solid Films 327-329, 854-856 (1998).

25 François, B., Pitois, O. \& François, J. Amphiphiles coordinated to block copolymers as a template for mesoporous materials. Adv. Mater. 7, 1041-1044 (1995). 
26 Karthaus, O., Maruyama, N., Cieren, X., Shimomura, M., Hasegawa, H. \& Hashimoto T. Water-assisted formation of micrometer-size honeycomb patterns of polymers. Langmuir 16, 6071-6076 (2000).

27 Yabu, H. \& Shimomura, M. Simple fabrication of micro lens arrays'. Langmuir 21 1709-1711 (2005).

28 Kojima, M., Hirai, Y., Yabu, H. \& Shimomura, M. The effects of interfacial tensions of amphiphilic copolymers on honeycomb-patterned films. Polym. J. 41, 667-671 (2009).

29 Yabu, H. \& Shimomura, M. Preparation of self-organized mesoscale polymer patterns on solid substrate: continuous pattern formation from receding meniscus. Adv. Func. Mater 14, 575-581 (2005).
30 Hirai, Y., Yabu, H., Matsuo, Y., Ijiro, K. \& Shimomura, M. 'Biomimetic anti-reflective and superhydrophobic silicon structures prepared by using self-organized honeycomb templates. J. Mater. Chem. 20, 10804-10808 (2010).

31 Fuard, D., Tzvetkova-Chevolleau, T., Decossas, S., Tracqui, P. \& Schiavone, P. Optimization of poly-di-methyl-siloxane (PDMS) substrates for studying cellular adhesion and motility. Microelectronic Eng. 85, 1289-1293 (2008).

32 Wang, D., Fujinami, S., Nakajima, K. \& Nishi, T. Investigation of reactive polymer polymer interface using nanomechanical mapping. Macromolecules 43, 3169-3172 (2010).

Supplementary Information accompanies the paper on Polymer Journal website (http://www.nature.com/pj) 